\title{
VALPROATE (VPA)-ASSOCIATED HEPATOTOXICITY
}

The metabolic pattern of VPA in 170 epileptic patients without symptoms or signs of hepatic side effects was compared to that in 11 patients with early symptoms and signs of hepatotoxicity and in 5 children with fulminant liver failure. A cohort of 470 patients in whom VPA serum metabolites had been measured were examined at several pediatric epilepsy clinics between 1986 and 1991 and results were evaluated at the Departments of Pediatrics, RittbergKrankenhaus; and Free University, Berlin, Germany; and University Hospital, Bern, Switzerland. In the reference group, infants receiving VPA monotherapy and comedication with other AEDs showed lower concentrations of the potential hepatotoxin 4-ene-VPA than did older children. Three of 4 children who died had massive lactic acidosis. In all children with probable VPA-associated hepatotoxicity, VPA metabolic patterns were different from the reference group, but were not specific or distinct, even in those who died. Increased values of 4-ene-VPA were found in only 1 of the 5 children with fulminant liver failure. The pattern of VPA metabolites did not distinguish between benign and life-threatening hepatic toxicities. (Siemes $\mathrm{H}$ et al. Valproate (VPA) metabolites in various clinical conditions of probable VPAassociated hepatotoxicity. Epilepsia March/April 1993; $\underline{34}$ : 332-346). (Reprints: Professor Dr Med Harmut Siemes, Rittberg-Kinderklinik, CarstennstraBe 58, D-1000 Berlin 45, Germany).

COMMENT. This most extensive study of VPA liver toxicity has shown that the primary toxic agent is most likely VPA itself and not the VPA metabolite, 4-ene-VPA. An inherited or acquired defect in the Boxidation of VPA may be contributory, and VPA may unmask an underlying metabolic disorder leading to mitochondrial dysfunction. (Appleton et al. Can I Neurol Sci 1990; 17:145). The initial symptoms and pattern of metabolites do not distinguish between those patients with a benign reversible liver dysfunction and those who may develop a fulminant and fatal liver failure. Young age, polypharmacy, status epilepticus, and febrile illness are factors associated with VPA toxicity.

\section{DESMOPRESSIN AND IMIPRAMINE-INDUCED CONVULSION}

A 10 year old boy with enuresis was admitted in coma to the Dumfries Royal Infirmary, Scotland. Treatment with intranasal desmopressin $40 \mathrm{mcg}$ nightly for 5 months had recently been supplemented with imipramine $25 \mathrm{mg}$. A generalized tonic seizure on admission was terminated by $5 \mathrm{mg}$ rectal diazepam and treatment for hyponatremia (serum sodium $113 \mathrm{mmol} / \mathrm{L}$ ). (Hamed $\mathrm{M}$ et al. Hyponatremic convulsion associated with desmopressin and imipramine treatment. BMI May 1 1993; $\underline{306}: 1169)$.

COMMENT. Avoid drug combination and limit fluids with desmopressin. 